IPHC-PHENO-09-02

LPSC 09-087

\title{
Transverse-momentum resummation for gaugino-pair production at hadron colliders
}

\author{
Jonathan Debove ${ }^{a}$, Benjamin Fuks $^{b}$, and Michael Klasen ${ }^{\text {* }}$ \\ a Laboratoire de Physique Subatomique et de Cosmologie, \\ Université Joseph Fourier/CNRS-IN2P3/INPG, 53 Avenue des Martyrs, F-38026 Grenoble, France \\ ${ }^{b}$ Institut Pluridisciplinaire Hubert Curien/Département Recherche Subatomiques, \\ Université de Strasbourg/CNRS-IN2P3, 23 Rue du Loess, F-67037 Strasbourg, France
}

(Dated: October 30, 2018)

\begin{abstract}
We present a first precision analysis of the transverse-momentum spectrum of gaugino pairs produced at the Tevatron and the LHC with center-of-mass energies of 1.96 and 10 or $14 \mathrm{TeV}$, respectively. Our calculation is based on a universal resummation formalism at next-to-leading logarithmic accuracy, which is consistently matched to the perturbative prediction at $\mathcal{O}\left(\alpha_{s}\right)$. Numerical results are given for the "gold-plated" associated production of neutralinos and charginos decaying into three charged leptons with missing transverse energy as well as for the pair production of neutralinos and charginos at two typical benchmark points in the constrained MSSM. We show that the matched resummation results differ considerably from the Monte Carlo predictions employed traditionally in experimental analyses and discuss the impact on the determination of SUSY mass parameters from derived transverse-mass spectra. We also investigate in detail the theoretical uncertainties coming from scale and parton-density function variations and non-perturbative effects.
\end{abstract}

\section{INTRODUCTION}

Weak-scale supersymmetry (SUSY) is a very well motivated extension of the Standard Model (SM) of particle physics. It can break the electroweak symmetry radiatively, allows for its grand unification with the local gauge symmetry of strong interactions, offers a natural explanation of the large hierarchy between electroweak and gravitational interactions, and appears naturally in string theories [1]. Among the new particles predicted by the Minimal Supersymmetric SM (MSSM), the fermionic partners of the neutral and charged gauge and Higgs bosons, called neutralinos and charginos, may be relatively light, and the lightest neutralino, stabilized by an at least approximate $R$-symmetry, represents one of the most promising dark matter candidates, whose gaugino/higgsino decomposition has important consequences for cosmology. The search for SUSY particles and the identification of their properties have thus become defining tasks of the current hadron collider program. Particular attention has since long been paid to the production of gauginos 2], which are produced either directly or through squark/gluino decays, decay themselves leptonically and are easily identifiable at the Tevatron [3] and at the LHC [4]. The Tevatron collaborations CDF and D0 have already published several SUSY searches in the "gold-plated" trilepton channel [3]. For the LHC, the CMS collaboration estimate the reach of the direct channel to be relatively modest (universal gaugino mass parameter $m_{1 / 2}<180 \mathrm{GeV}$ ) and relevant only for scenarios with heavy colored particles (universal scalar masses $\left.m_{0}>1000 \mathrm{GeV}\right)$. However, the ATLAS collaboration have shown that a discovery can be made in this channel for a wider range of gaugino masses $\left(m_{1 / 2} \leq 360 \mathrm{GeV}\right)$ and independently of $m_{0}$ already with a low luminosity of a few $\mathrm{fb}^{-1}[4]$. Gaugino pair production is therefore a very important SUSY discovery channel at both currently running hadron colliders.

For an efficient suppression of the SM background from vector-boson and top-quark production and a precise determination of the underlying SUSY-breaking model and masses, an accurate theoretical calculation of the signal (and background) cross section is imperative. As the lightest SUSY particle (LSP) escapes undetected, the key distribution for SUSY discovery and measurements is the missing transverse-energy $\left(E_{T}\right)$ spectrum, which is typically restricted by a cut of $20 \mathrm{GeV}$ at the Tevatron and $30 \mathrm{GeV}$ at the LHC. While the SUSY particle pair is produced with zero transverse momentum $\left(p_{T}\right)$ in the Born approximation, the possible radiation of gluons from the quark-antiquark initial state or the splitting of gluons into quark-antiquark pairs at $\mathcal{O}\left(\alpha_{s}\right)$ in the strong coupling constant induces transverse momenta extending to quite substantial values and must therefore be taken into account [5]. In addition, the perturbative calculation diverges at small $p_{T}$, indicating the need for a resummation of soft-gluon radiation to all orders [6]. Only after a consistent matching of the perturbative and resummed calculations an accurate description of the (missing) transverse energy spectrum and precise measurements of the SUSY particle masses can be achieved.

In this Letter, we report on the first precision analysis of the transverse-momentum spectrum of gaugino pairs produced at the Tevatron and the LHC with center-ofmass energies of 1.96 and 10 or $14 \mathrm{TeV}$, respectively. We briefly describe in the following section our implementation of the resummation formalism, which has been improved with respect to the original proposal in numerous respects, and present then numerical results for the production of various gaugino pairs at two typical MSSM benchmark points. We also discuss the impact of the computed precise transverse-momentum spectrum on the determination of SUSY mass parameters and in- 
vestigate in detail the remaining theoretical uncertainties coming from scale and parton-density function variations and non-perturbative effects.

\section{TRANSVERSE-MOMENTUM RESUMMATION}

In the Born approximation, the production of neutralinos and charginos at hadron colliders

$$
p \bar{p}, p p \rightarrow q \bar{q}^{\prime}+X \rightarrow \tilde{\chi}_{i} \tilde{\chi}_{j}+X
$$

is induced by the quarks $q$ and antiquarks $\bar{q}^{\prime}$ in the initial (anti-)protons and is mediated by $s$-channel electroweak gauge-boson and $t$ - and $u$-channel squark exchanges. Its partonic cross section $\sigma^{(0)}$ can be expressed in terms of the gaugino and squark masses $m_{\tilde{\chi}_{i, j}^{0, \pm}}$ and $m_{\tilde{q}}$, the masses of the electroweak gauge bosons, the Mandelstam variables $s, t$ and $u$, and generalized charges [2].

At leading order (LO) in the strong coupling constant, $\mathcal{O}\left(\alpha_{s}\right)$, virtual loop and real parton emission corrections must be taken into account [5]. The latter induce transverse momenta of the gaugino pair, that extend typically to values of the order of the gaugino mass. In the small$p_{T}$ region, where the bulk of the events is produced, the convergence of the perturbative expansion is spoiled due to the presence of large logarithms $\alpha_{s}^{n} / p_{T}^{2} \ln ^{m}\left(M^{2} / p_{T}^{2}\right)$ with $m \leq 2 n-1$. These must be resummed to all orders in impact parameter $(b)$ space,

$$
\frac{\mathrm{d} \sigma^{\mathrm{RES}}}{\mathrm{d} p_{T}^{2}}\left(p_{T}, M, s\right)=\frac{M^{2}}{s} \int_{0}^{\infty} \mathrm{d} b \frac{b}{2} J_{0}\left(b p_{T}\right) \mathcal{W}(b, M, s),
$$

in order to correctly implement transverse-momentum conservation. Here, $b$ describes the minimal distance of the two incident particles in the limit of no interaction and is the conjugate variable of the transverse momentum $p_{T} ; J_{0}(x)$ is the $0^{\text {th }}$-order Bessel function; $M$ is the invariant mass of the gaugino pair; and Eq. (2) is evaluated numerically as in Ref. [7]. After a Mellin transform with respect to $z=M^{2} / s$, the hadronic cross section simplifies from a convolution to a product of the parton densities evaluated at the factorization scale $\mu_{F}$ with the partonic cross section, the $N$-moment of the latter

$$
\mathcal{W}_{N}(b, M)=\mathcal{H}_{N}(M) \exp \left[\mathcal{G}_{N}(L)\right]
$$

factorizing further into a $b$-independent function

$$
\mathcal{H}_{N}(M)=\sigma^{(0)}(M)\left[1+\frac{\alpha_{s}}{\pi} \mathcal{H}_{N}^{(1)}(M / Q)+\ldots\right],
$$

that is therefore finite as $p_{T} \rightarrow 0$ or $b \rightarrow \infty$, and an exponential form factor

$$
\mathcal{G}_{N}(L)=L g^{(1)}\left(\alpha_{s} L\right)+g^{(2)}\left(\alpha_{s} L\right)+\ldots,
$$

which resums the divergent leading and next-to-leading contributions in the logarithm $L=\ln \left(Q^{2} b^{2} / b_{0}^{2}\right)$ through the process-independent functions $g^{(1,2)}$. While $b_{0}=$ $2 e^{-\gamma_{E}}$ is of kinematical origin, the scale $Q \sim M$ accounts for the arbitrary separation of the two factors. Unphysical logarithmic divergences at $b \rightarrow 0$ are regularized by replacing $L$ with $\tilde{L}=\ln \left(Q^{2} b^{2} / b_{0}^{2}+1\right)$. The evolution from the factorization scale $\mu_{F}$ and the low scale $b_{0} / b$ to the high scale $Q$ is performed in $\mathcal{H}_{N}(M)$ and $\exp \left[\mathcal{G}_{N}\right]$, respectively, leaving the latter $\mu_{F}$-independent and allowing for a convolution of the partonic cross section with the parton densities in the (anti-)protons at $\mu_{F}$ [7].

Although SUSY particles appearing in the virtual corrections must be taken into account for a proper renormalization of ultraviolet divergences, their heavy masses are known to leave only little imprint numerically. In particular, the ratios of NLO/LO total cross sections vary only from 1.26-1.28 at the Tevatron and 1.24-1.28 at the LHC for squark masses of $350-1000 \mathrm{GeV}$ (for the similar case of sleptons, see also Fig. 4(a) in Beenakker, Klasen et al. [5]). The restoration of the equality of the weak scalar $(\hat{g})$ and vector gauge couplings $(g)$ furthermore requires the introduction of a finite SUSY-restoring counter term, $\hat{g}=g\left[1-\alpha_{s} /(6 \pi)\right]$. Finally, quark-gluon initial states can induce the production of gauginos in association with real squarks decaying into quarks and gauginos. The singularity associated with the pole of the on-shell squark propagator is regularized by a small finite width $\Gamma_{\tilde{q}} \sim 10^{-2} m_{\tilde{q}}$ (the exact value has little influence numerically), and the on-shell contribution is subtracted from the total cross section to avoid double-counting $[5,8]$.

\section{NUMERICAL RESULTS}

To obtain a valid hadronic cross section at all values of $p_{T}$, the $\mathcal{O}\left(\alpha_{s}\right)$ (LO) and resummed (RES) partonic cross sections are matched by subtracting from their sum the perturbatively expanded (EXP) resummed cross section,

$$
\frac{\mathrm{d} \sigma}{\mathrm{d} p_{T}^{2}}=\frac{\mathrm{d} \sigma^{\mathrm{LO}}}{\mathrm{d} p_{T}^{2}}+\left[\frac{\mathrm{d} \sigma^{\mathrm{RES}}}{\mathrm{d} p_{T}^{2}}-\frac{\mathrm{d} \sigma^{\mathrm{EXP}}}{\mathrm{d} p_{T}^{2}}\right]
$$

and by performing numerically the necessary inverse Mellin and Fourier transforms, kinematic integrations, and parton density convolutions. The parton densities are evaluated in the most recent parameterization by the CTEQ collaboration CTEQ6.6M [9] with $\mu_{F}$ (and the renormalization scale $\mu_{R}$ ) set to the average mass $\bar{m}$ of the final state particles. The SUSY particle masses are obtained from universal parameters defined at the grand unification scale through the renormalization group running implemented in the computer code SUSPECT2.3 [10].

To be specific, we choose two minimal supergravity benchmark points. The first is the low-mass point LM0 (SU4) with universal fermion mass $m_{1 / 2}=160 \mathrm{GeV}$, scalar mass $m_{0}=200 \mathrm{GeV}$, trilinear coupling $A_{0}=-400$ $\mathrm{GeV}$, bilinear Higgs mass parameter $\mu>0$, and ratio of 
Higgs vacuum expectation values $\tan \beta=10$ [4]. It has been been defined by the CMS (ATLAS) collaboration with the objective of high cross sections and thus early discovery at the LHC, as the resulting gaugino, squark and slepton masses $m_{\tilde{\chi}_{2}^{0}}=m_{\tilde{\chi}_{1}^{ \pm}}=113 \mathrm{GeV}, m_{\tilde{\chi}_{1}^{0}}=61$ $\mathrm{GeV}, m_{\tilde{q}} \simeq 420 \mathrm{GeV}$, and $m_{\tilde{l}} \simeq 220 \mathrm{GeV}$ lie just beyond the current Tevatron limits. In this scenario, the lightest chargino and second-lightest neutralino decay with $35 \%$ and 15\% probability through virtual sleptons to the LSP and one and two charged leptons, respectively [11].

Our second benchmark point is the Snowmass point (and slope, SPS) 1a' with $m_{1 / 2}=250 \mathrm{GeV}, m_{0}=70$ $\mathrm{GeV}, A_{0}=-300 \mathrm{GeV}, \mu>0$, and $\tan \beta=10$, which is similar to the point LM1 (SU1) defined by the CMS (ATLAS) collaboration and, with its SUSY particle masses of $m_{\tilde{\chi}_{2}^{0}}=m_{\tilde{\chi}_{1}^{ \pm}}=183 \mathrm{GeV}, m_{\tilde{\chi}_{1}^{0}}=98 \mathrm{GeV}, m_{\tilde{q}} \simeq 550 \mathrm{GeV}$, and $m_{\tilde{l}}=120 \ldots 190 \mathrm{GeV}$, is known to be compatible with all high-energy mass bounds and low-energy precision data. Here, the lightest chargino and second-lightest neutralino decay almost exclusively to three charged leptons and missing transverse energy, albeit through real sleptons, which may be experimentally reconstructed through endpoints in kinematic distributions [12].

In Fig. 1, we show the corresponding transversemomentum spectra of chargino-neutralino pairs produced at run II of the Tevatron (top) and the initial run of the LHC (bottom) with center-of-mass energies of $\sqrt{S}=1.96$ and $10 \mathrm{TeV}$, respectively. As expected, the LO predictions (dashed curves) diverge at low $p_{T}$, but become finite after matching them to the resummed predictions at next-to-leading logarithmic (NLL) accuracy (full curves). In this region, the perturbative expansions of the resummed predictions (dots) coincide with those at LO, while at large $p_{T}$ they coincide with the resummed ones. Through resummation, the perturbative predictions are considerably enhanced even at values of $p_{T}$, which are of the order of the experimental $E_{T}$ cuts. It is therefore important to clearly distinguish the effects induced by QCD radiation and by the unobserved LSPs and neutrinos. By construction, the matched LO+NLL prediction allows to reproduce the correct $\mathcal{O}\left(\alpha_{s}\right)$ correction $(K)$ factor of the total perturbative cross section after integration over $p_{T}$, e.g. of 1.26 at the Tevatron. For comparison, we also show the matched LO+NLL $p_{T}$-spectrum (dotdashed curve) for the $14 \mathrm{TeV}$ design energy of the LHC, which extends to considerably larger values of $p_{T}$ than at $10 \mathrm{TeV}$. The theoretical predictions are influenced by three main sources of uncertainty: scale variations, evaluated in the canonical range of $\mu_{F, R} / \bar{m}=0.5 \ldots 2$ (shaded bands), variations of the parton densities, evaluated through $\Delta_{\mathrm{PDF}}=\sqrt{\sum_{i=1}^{22}\left(\mathrm{~d} \sigma_{i}^{+}-\mathrm{d} \sigma_{i}^{-}\right)^{2}} /(2 \mathrm{~d} \sigma)$ along the 22 eigenvector directions defined by the CTEQ collaboration (lower curves), and three choices of non-perturbative (NP) form factors, evaluated through $\Delta_{\mathrm{NP}}=\left(\mathrm{d} \sigma^{\mathrm{NP}}-\right.$ $\mathrm{d} \sigma) / \mathrm{d} \sigma$ (inserts) [13]. For $p_{T}>5 \mathrm{GeV}$, all theoretical
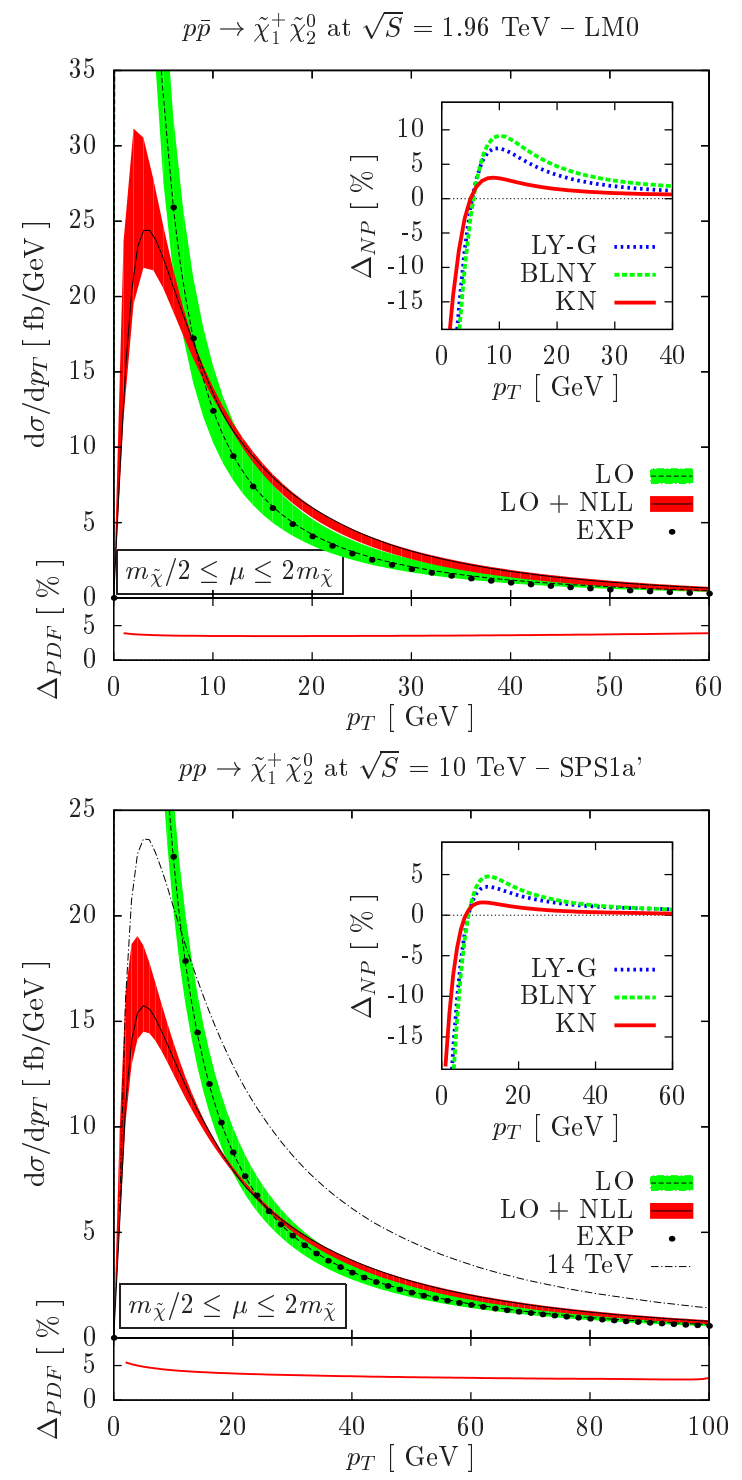

FIG. 1: Transverse-momentum spectra of chargino-neutralino pairs at the Tevatron (top) and the LHC (bottom). The LO calculation (green/dashed) is matched to the resummed calculation (red/full) by subtracting its fixed-order expansion (dotted). The scale uncertainty is shown as a shaded band, the PDF (below) and non-perturbative (insert) uncertainties as separate graphs, and the matched result for the LHC design energy of $\sqrt{S}=14 \mathrm{TeV}$ as a dot-dashed line (bottom).

uncertainties are smaller than $5 \%$ for the LO+NLL predictions. In particular, the $5 \% \mathrm{PDF}$ uncertainty is similar to the one obtained for weak boson production [9].

In Fig. 2, the matched $p_{T}$-spectra for chargino (dotted) and neutralino (dot-dashed) pairs are compared to those of the tri-lepton channel (full/dashed) discussed above. While positive and negative charginos are produced with equal rates in $p \bar{p}$ collisions at the Tevatron, their rates differ slightly in $p p$ collisions at the LHC. The cross sections for neutralino pair production are about one order 


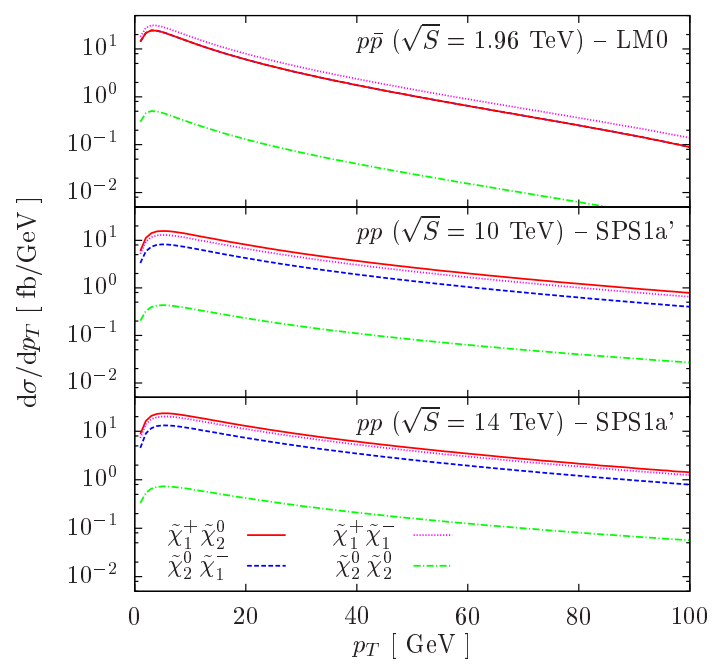

FIG. 2: Transverse-momentum spectra at LO+NLL for the associated production of charginos and neutralinos (full and dashed) as well as chargino (dotted) and neutralino (dotdashed) pairs (dotted) in three different collider modes.

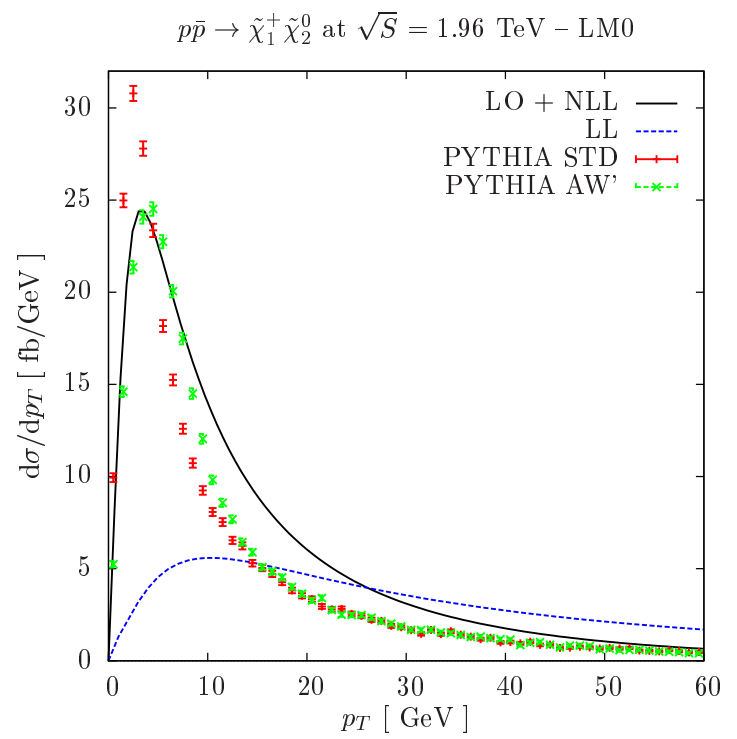

FIG. 3: Transverse-momentum spectra at LO+NLL (full), LL (dashed), and generated by the PYTHIA parton shower with default (bars) and tuned (crosses) parameters at the Tevatron.

of magnitude smaller, as the second-lightest neutralino couples to the $s$-channel $Z^{0}$-boson only through its relatively small Higgsino component.

In experimental analyses, QCD radiation in hadronic collisions is usually simulated with tree-level matrix elements and parton showers based on an exponential Sudakov form factor, which resums the leading logarithms (LL) and some next-to-leading logarithms. In Fig. 3 , we compare therefore our matched $\mathrm{LO}+\mathrm{NLL}$ prediction (full curve) with our resummed prediction at LL order (dashed curve) and the default (bars) and tuned (crosses) predictions of the PYTHIA6.4 Monte Carlo (MC) generator [14]. While the default MC prediction is clearly improved beyond the LL approximation and approaches the LO+NLL result, it peaks at too small values of $p_{T}$. Tuning the intrinsic $p_{T}$ of the partons in the proton to 2.1 $\mathrm{GeV}$ for $Z^{0}$-bosons (CDF tune $\mathrm{AW}$ ) and $4 \mathrm{GeV}$ for gaugino pairs (our tune AW') improves the description of the peak, but still underestimates the intermediate $p_{T}$-region and the mean value of $p_{T}(14 \mathrm{GeV}$ for PYTHIA6 . 4, $15 \mathrm{GeV}$ for our tune AW', and $18 \mathrm{GeV}$ for our LO+NLL prediction). This has, of course, a direct impact on the determination of the gaugino (and slepton) masses through variables derived from the transverse momenta of the observed leptons $p_{T, i}$ and $\mathbb{E}_{T}$, such as the effective mass $M_{\mathrm{eff}}=\sum_{i} p_{T, i}+E_{T}$ [15] or the stransverse mass [16]. A detailed discussion of signal and background distributions in these variables, including also the experimental resolution, is beyond the scope of this work. Let us mention, however, that the contribution of unmeasured (low$p_{T}$ or forward) or mismeasured hadronic energy to the "fake" $E_{T}$ is under close scrutiny both at the Tevatron and at the LHC. The ATLAS trilepton analysis, e.g., does not identify jets with $p_{T}<10 \mathrm{GeV}$, and an optional cut on jets with $p_{T}>20 \mathrm{GeV}$ reduces the significance considerably. As the two LSPs are often back-to-back, the $E_{T}$ in the trilepton analysis is required to be relatively small $(>30 \mathrm{GeV})$. It can then be affected by an error of up to $10 \%$ (Aad et al. [4]).

\section{CONCLUSION}

In summary, we have calculated the transversemomentum spectrum of gaugino pairs produced at hadron colliders at next-to-leading logarithmic accuracy. We have demonstrated that this renders the perturbative prediction finite, modifies considerably the traditionally used Monte Carlo predictions, and reduces the remaining theoretical uncertainties to the level of $5 \%$. We have also briefly discussed the impact of our calculation on the experimental determination of the gaugino masses, but leave a detailed experimental simulation to future work.

We thank B. Clément for useful discussions. This work has been supported by a Ph.D. fellowship of the French ministry for education and research and by the TheoryLHC-France initiative of the CNRS/IN2P3.

* klasen@lpsc.in2p3.fr

[1] H. P. Nilles, Phys. Rept. 110, 1 (1984); H. E. Haber and G. L. Kane, Phys. Rept. 117, 75 (1985).

[2] V. Barger, R. Robinett, W. Keung and R. Phillips, Phys. Lett. B 131, 372 (1983); S. Dawson, E. Eichten and C. Quigg, Phys. Rev. D 31, 1581 (1985); G. Bozzi, 
B. Fuks, B. Herrmann and M. Klasen, Nucl. Phys. B 787, 1 (2007); J. Debove, B. Fuks and M. Klasen, Phys. Rev. D 78, 074020 (2008); B. Fuks, B. Herrmann and M. Klasen, Nucl. Phys. B 810, 266 (2009).

[3] T. Aaltonen et al. [CDF Collaboration], Phys. Rev. Lett. 101, 251801 (2008); V. M. Abazov et al. [D0 Collaboration], arXiv:0901.0646 [hep-ex].

[4] G. Aad et al. [ATLAS Collaboration], arXiv:0901.0512, in particular pp. 368-396 and 1643-1659; G. Bayatian et al. [CMS Collaboration], J. Phys. G 34, 995 (2007), in particular Sec. 13.14.

[5] W. Beenakker, R. Höpker, M. Spira and P. Zerwas, Nucl. Phys. B 492, 51 (1997); W. Beenakker, M. Krämer, T. Plehn, M. Spira and P. Zerwas, Nucl. Phys. B 515, 3 (1998); E. Berger, M. Klasen and T. Tait, Phys. Rev. D 59, 074024 (1999); W. Beenakker, M. Klasen, M. Krämer, T. Plehn, M. Spira and P. Zerwas, Phys. Rev. Lett. 83, 3780 (1999); E. Berger, M. Klasen and T. Tait, Phys. Lett. B 459, 165 (1999); E. Berger, M. Klasen and T. Tait, Phys. Rev. D 62, 095014 (2000).

[6] G. Bozzi, B. Fuks and M. Klasen, Phys. Rev. D 74, 015001 (2006); G. Bozzi, B. Fuks and M. Klasen, Nucl. Phys. B 777, 157 (2007); G. Bozzi, B. Fuks and M. Klasen, Nucl. Phys. B 794, 46 (2008); B. Fuks, M. Klasen, F. Ledroit, Q. Li and J. Morel, Nucl. Phys.
B 797, 322 (2008).

[7] G. Bozzi, S. Catani, D. de Florian and M. Grazzini, Nucl. Phys. B 737, 73 (2006).

[8] C. S. Li, Z. Li, R. J. Oakes and L. L. Yang, Phys. Rev. D 77, 034010 (2008).

[9] P. M. Nadolsky et al., Phys. Rev. D 78, 013004 (2008).

[10] A. Djouadi, J. L. Kneur and G. Moultaka, Comput. Phys. Commun. 176, 426 (2007).

[11] M. Mühlleitner, A. Djouadi and Y. Mambrini, Comput. Phys. Commun. 168, 46 (2005).

[12] J. A. Aguilar-Saavedra et al., Eur. Phys. J. C 46, 43 (2006).

[13] G. A. Ladinsky and C. P. Yuan, Phys. Rev. D 50, 4239 (1994); F. Landry, R. Brock, P. M. Nadolsky and C. P. Yuan, Phys. Rev. D 67, 073016 (2003); A. V. Konychev and P. M. Nadolsky, Phys. Lett. B 633, 710 (2006).

[14] T. Sjöstrand, S. Mrenna and P. Skands, JHEP 0605, 026 (2006); R. Field [CDF Collaboration], FERMILABPUB-06-408-E (2006).

[15] I. Hinchliffe, F. E. Paige, M. D. Shapiro, J. Soderqvist and W. Yao, Phys. Rev. D 55, 5520 (1997).

[16] C. G. Lester and D. J. Summers, Phys. Lett. B 463, 99 (1999). 